\title{
Cost-Effectiveness of Comprehensive Medication Reviews Versus Noncomprehensive Medication Review Interventions and Subsequent Successful Medication Changes in a Medicare Part D Population
}

\author{
Chanadda Chinthammit, BSPharm, MS; Edward P. Armstrong, PharmD; Kevin Boesen, PharmD; \\ Rose Martin, PharmD; Ann M. Taylor, MPH, MCHES; and Terri Warholak, RPh, PhD
}

\begin{abstract}
BACKGROUND: An estimated 1.5 million preventable medication-related adverse events occur annually, with some resulting in serious injury and even death. To help address this issue, the Centers for Medicare \& Medicaid Services (CMS) now require medication therapy management (MTM) programs to offer comprehensive medication reviews (CMRs) to all Medicare Part $D$ beneficiaries at least once a year. During a CMR, patients receive an extensive amount of medication and educational information. In contrast, noncomprehensive medication reviews (non-CMRs) are more targeted and focus on resolving a particular medication-related problem (MRP) via short patient consultations, patient letters, and direct provider interventions.
\end{abstract}

OBJECTIVE: To conduct a cost-effectiveness analysis comparing CMRs with non-CMR interventions on successful medication regimen changes and reductions in adverse drug events (ADEs).

METHODS: This decision analytic model compared the cost-effectiveness of CMRs with other intervention methods (non-CMRs) from a payer's perspective. For this model, a successful outcome was defined as a beneficiary case devoid of an ADE due to MRPs. The model was extensively tested and subjected to a thorough one-way sensitivity analysis and a second-order probabilistic sensitivity analysis with 10,000 iterations from the variable distributions.

RESULTS: Non-CMR interventions were less costly and more effective than CMRs. The point estimate for direct medical costs was $\$ 193$ for CMRs and $\$ 157$ for non-CMRs, and the estimated probability of avoiding an ADE was 0.93 and 0.94 for CMRs and non-CMRs, respectively. The 10,000 iterationMonte Carlo simulation scatterplot and cost-effectiveness acceptability curve (CEAC) revealed a dominance by non-CMRs in preventing harmful ADEs from cost and effectiveness perspectives; however, there was an overlap in the $95 \%$ Cls for both cost and ADEs prevented. Despite this, a non-CMR intervention saved estimated $\$ 5,377.08$ per ADE prevented. Oneway sensitivity analysis indicated the results were sensitive to the cost of treating a preventable ADE. In $100 \%$ of cases, the CEAC demonstrated that non-CMRs were likely the most cost-effective intervention regardless of the health plan's willingness to pay.

CONCLUSIONS: The cost-effectiveness acceptability curve suggests that non-CMR interventions were less costly and more effective than CMRs; however, there was overlap in the $95 \%$ Cls for costs and ADEs prevented. In all cases, the CEAC demonstrated that non-CMRs were the most economical intervention with regard to time and cost. Non-CMRs show promise as a viable method to address MRPs, reduce ADEs, and improve patient-related health outcomes.

J Manag Care Spec Pharm. 2015;21(5):381-89

Copyright $\odot 2015$, Academy of Managed Care Pharmacy. All rights reserved.

\section{What is already known about this subject}

Medication-related problems (MRPs) are frequent and critical problems in the United States, with some leading to harmful adverse drug events (ADEs) and subsequent medical costs.

Potential MRPs identified during a targeted medical review (TMR) may be addressed via comprehensive medication reviews (CMRs) or more targeted means such as short patient consultations, patient letters, and direct provider interventions (nonCMRs).

TMRs and CMRs are required for Medicare Part D beneficiaries who meet minimum qualification criteria, yet the Centers for Medicare \& Medicaid Services (CMS) use only the annual percentage of patients who receive a CMR as a performance metric or star rating for medication therapy management programs.

\section{What this study adds}

This study provides a cost-effectiveness analysis comparing CMRs with non-CMR interventions on successful medication regimen changes and reductions in ADEs from a payer's perspective. Study results suggest that non-CMR interventions are more effective and less costly than CMRs.

Important new information is provided regarding non-CMR interventions that suggests the need for consideration and integration of these interventions into the CMS star ratings program.

$\mathrm{M}$ edication-related errors are frequent and important problems in the United States. ${ }^{1}$ An estimated 1.5 mil-

lion preventable medication-related adverse events occur each year, with some leading to serious injury and even death. ${ }^{2}$ Adverse drug events (ADEs) are associated with longer hospitalizations (e.g., 8 to 12 days) and higher hospital costs (e.g., $\$ 16,000$ to $\$ 24,000$ per patient), ${ }^{2}$ resulting in an additional $\$ 177$ billion in medication-related morbidity and mortality. ${ }^{3}$ Furthermore, using multiple medications increases the risk of experiencing an ADE. ${ }^{4-7}$ Medication mismanagement and nonadherence are also common causes of medicationrelated problems (MRPs) and ADEs, ${ }^{8}$ and poor medication adherence (i.e., nonadherence) is associated with 33\%-69\% of medication-related hospital admissions. ${ }^{9}$ Patients with low 
health literacy are at higher risk for adverse outcomes due to misunderstandings, ${ }^{10}$ and increased age may be an independent risk factor for medication errors and ADEs. ${ }^{11,12}$ Despite these challenges, studies show that clinical pharmacists can effectively detect and prevent critical MRPs. In particular, 41\%$96 \%$ of pharmacist recommendations are accepted by prescribers. ${ }^{13-17}$ Finally, pharmacist-led discharge counseling contributes to better clinical outcomes (e.g., fewer heart failure events and nonfatal stroke) and improved economic outcomes. ${ }^{18-20}$

The role of the pharmacist as a key partner on an interprofessional health care team has drawn much public attention with the inclusion of medication therapy management (MTM) services as part of the Medicare Modernization Act of 2003. ${ }^{21}$ While other health care providers can provide MTM services, pharmacists receive extensive training in pharmacology, medicinal chemistry, pharmacotherapy, pharmacokinetics, and drug-drug interactions, which equips them with the skills and knowledge to solve drug-related problems (DRPs; also known as medication-related problems [MRPs]) and implement treatment solutions. ${ }^{22}$

MTM comprises a broad range of services and intervention strategies that involve pharmacists working in tandem with other health care providers, most notably physicians. ${ }^{23-25}$ In general, MTM services provide a variety of medication therapy protocols, depending on the type of service center-some programs provide face-to-face consultation while others utilize phone consultation.

As part of MTM services, the Centers for Medicare \& Medicaid Services (CMS) mandate that all programs offer comprehensive medication reviews (CMRs) to eligible beneficiaries at least once a year. As defined by CMS, a CMR is a detailed comprehensive review of a beneficiary's medications and chronic conditions aimed at optimizing patient outcomes. CMRs require extensive patient consultations with additional written follow-up, which is a time-consuming task for pharmacists and patients. During a CMR, a large amount of complex information is provided to patients, some of whom may have limited health literacy ${ }^{26} \mathrm{CMS}$ also requires targeted medication reviews (TMRs) for ongoing medication monitoring at least quarterly, based on beneficiary eligibility. A TMR is defined as a review that assesses medication use (e.g., beta2-agonists are recommended for patients with acute symptoms), monitoring for DRPs, or other unresolved issues. ${ }^{27}$ MTM programs can also utilize non-CMR interventions such as brief targeted consultations, patient letters, and direct provider interventions to address DRPs and optimize patient outcomes. In contrast, nonCMR interventions are more succinct, are specific to DRPs, and can be less intimidating for patients. ${ }^{26}$ Interestingly, prescribers are more likely to approve recommended medication changes resulting from targeted interventions (non-CMRs). ${ }^{26}$ In general, MTM programs seldom use non-CMR interventions, since CMS evaluates program performance based on the percentage of patients annually who receive a CMR.
Recent studies reporting the benefits of MTM services have gained attention from insurers, payers, providers, and policymakers given that these services may have a positive impact on clinical, economic, and humanistic outcomes. ${ }^{28-32}$ Additionally, several studies assert that MTM services are cost-effective and provide a positive return on investment (ROI) ${ }^{30,33,34}$ One study reported that a $\$ 1$ expenditure resulted in a $\$ 12$ ROI. ${ }^{30}$ Although a plethora of research studies exists on the clinical and economic benefits of MTM, there is a dearth of studies comparing the benefit of CMRs with non-CMR interventions. Thus, the purpose of this study was to compare the cost-effectiveness of CMRs with non-CMR interventions at a large MTM program providing telephone-based services.

\section{Methods}

\section{The Model}

This study was a cost-effectiveness evaluation of the Medication Management Center (MMC) program at the University of Arizona College of Pharmacy, which provides MTM services to 10 million patients-both Medicare and private insurance beneficiaries-throughout the United States, using a centralized call center approach. The cost-effectiveness model compared CMRs and non-CMR interventions in Medicare Part D patients who (a) were automatically enrolled into the MTM because they met all of CMS requirements in 2012, including having at least 3 chronic health conditions, taking at least 3 medications, and incurring costs for medication greater than $\$ 3,100$ annually, ${ }^{35}$ and (b) had at least 1 MRP (e.g., adjustment of treatment regimen, potential drug interaction, or untreated indication).

Once an MRP was detected, the MMC staff (pharmacists, pharmacy interns, and pharmacy technicians) made telephone calls to the patient to conduct a CMR. If the patient agreed, he or she was included in the CMR intervention group. If the patient did not receive a CMR (i.e., not interested in receiving the CMR or unreachable), that patient was included in the non-CMR intervention group. MMC staff made recommendations to prescribers to resolve the identified drug therapy issues. A successful recommendation change was defined as an expected medication change identified in subsequent pharmacy claims. ${ }^{26}$ For the model, successful outcomes included (a) a change in therapy resulting from a CMR or non-CMR or (b) no change in therapy from a CMR or non-CMR and no documented ADE. A change in therapy resulting from a CMR or non-CMR was included as an outcome, since it served as a reasonable proxy to long-term clinical outcomes that were not available in pharmacy claims data. No change in therapy from a CMR or non-CMR and no documented ADE were included as an outcome based on the assumption that other causes of ADEs besides MRPs would be very uncommon. On the other hand, unresolved MRPs could lead to ADEs and, in some cases, might require additional health care resource utilization such as hospitalization and/or emergency department visits 


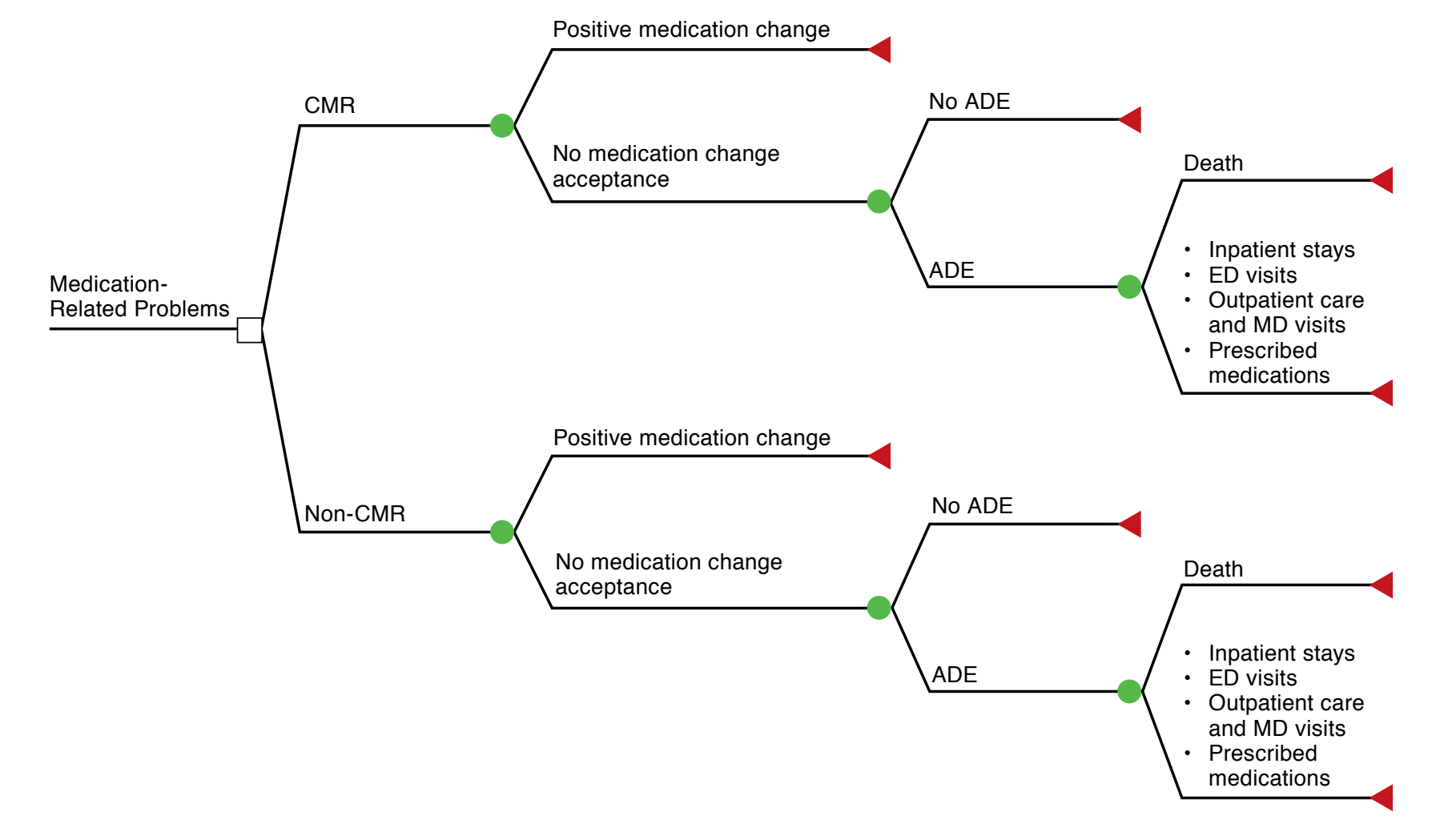

$A D E=$ adverse drug event $C M R=$ comprehensive medication review; $E D=$ emergency department; non- $C M R=$ noncomprehensive medication review; $M D=$ physician .

(Figure 1). 3,36,37 Given the unavailability of data to suggest otherwise, we assumed that medication adherence rates were similar in the CMR and non-CMR groups; therefore, this adherence variable was excluded from the model. The perspective of a health care payer was chosen with a 1-year time period, since we used data from a study investigating ADE costs incurred in 1 year. ${ }^{38}$ Thus, discounting was not required.

A systematic review of published ADE-related research was conducted to identify probabilities associated costs to populate the model. The literature searches in PubMed and Google Scholar were conducted between October 2013 and February 2014. The Boolean operator "AND" was used to identify studies describing the relationship between MRPs and harmful ADEs that require additional health care services for treatment. The first theme was MRPs, identified by the consolidation of Medical Subject Heading (MeSH) terms drug-related side effects and adverse reactions OR adverse drug reaction reporting systems OR medication error OR text words DRP* OR MRP* OR error*. The second theme was harmful consequences of MRPs, identified by the consolidation of MeSH terms hospitalization OR emergency service, hospital OR text words admission* OR ER*. From these studies, the probability of harmful ADEs due to
MRPs was identified (Table 1). In order to obtain average costs associated with ADEs due to MRPs, the first search term mentioned was combined with another theme, health care expenditure, which was identified by consolidation of MeSH terms costs and cost analysis OR text words costs* OR cost-of-illness* (Table 1). We considered articles published only in English and those that were original studies or observational studies for inclusion. References obtained from the retrieved studies were also searched for additional articles. Titles and abstracts were screened, followed by full-text review.

\section{Cost-Effective Model Inputs}

MRPs and Successful Medication Changes. We used unpublished quality improvement data to assess the impact of CMRs and non-CMR interventions on MRPs and positive medication changes to populate the event probabilities in the model. ${ }^{26}$ These probabilities were obtained from a sample size of more than 400,000 beneficiaries in which 288,701 had MRPs. These de-identified beneficiary data were obtained from the MMC's Medicare Part D 2012 CMS annual report, which contained summary information for each beneficiary enrolled in the center's MTM program. In this sample, $12.2 \%$ of beneficiaries were 


\section{TABLE 1 Parameters Utilized in the Economic Model}

\begin{tabular}{|c|c|c|c|}
\hline Model Parameter & Base Case & Distribution/Range & Source of Data/Comments \\
\hline \multicolumn{4}{|l|}{ Probabilities } \\
\hline $\begin{array}{l}\text { Probability of medication change } \\
\text { according to CMR recommendation }\end{array}$ & 0.28 & $\begin{array}{l}\text { Beta distribution range } \\
\text { (min-max): } 0.27-0.29\end{array}$ & Buhl et al. ${ }^{26}$ \\
\hline $\begin{array}{l}\text { Probability of medication change } \\
\text { according to non-CMR recommendation }\end{array}$ & 0.35 & $\begin{array}{l}\text { Beta distribution range } \\
\text { (min-max): } 0.34-0.35\end{array}$ & Buhl et al. 26 \\
\hline $\begin{array}{l}\text { Probability of having harmful ADE due } \\
\text { to MRP }\end{array}$ & 0.09 & $\begin{array}{l}\text { Beta distribution range } \\
\text { (min-max): } 0.05-0.15\end{array}$ & Kuo et al. ${ }^{36}$ \\
\hline Probability of death due to ADE & 0.02 & $\begin{array}{l}\text { Beta distribution range } \\
\text { (min-max): } 3.46 \times 10^{-6}-0.21\end{array}$ & Kuo et al. ${ }^{36}$ \\
\hline \multicolumn{4}{|l|}{ Costs } \\
\hline $\begin{array}{l}\text { Incremental costs of providing CMRs } \\
\text { (over non-CMR interventions) }\end{array}$ & $\$ 15.02$ & $\begin{array}{l}\text { Gamma distribution range } \\
\text { (min-max): } \$ 2.64-\$ 52.80\end{array}$ & $\begin{array}{l}\text { Based on extra time required to perform CMRs (over non-CMR } \\
\text { interventions) per prescription (average time }=10.13 \text { minutes, } \\
\mathrm{SD}=5.31 \text { minutes) }\end{array}$ \\
\hline Cost of treating a preventable ADE & $\$ 2,643.93$ & $\begin{array}{l}\text { Gamma distribution range } \\
\text { (min-max): } \$ 116.87-\$ 10,056.44\end{array}$ & $\begin{array}{l}\text { Field et al., adjusted to } 2012 \text { numbers by using Consumer Price } \\
\text { Index } 38,44\end{array}$ \\
\hline
\end{tabular}

interested in receiving CMRs; therefore, they were classified in the CMR group. Those beneficiaries in the CMR group had an average age of 73 years (standard deviation $[S D]=10$ years). Those beneficiaries in the non-CMR group ( $87.7 \%$ of those in the sample) had an average age of 75 years ( $\mathrm{SD}=10$ years). Non-CMR patients with MRPs were more likely to have a successful medication change resulting from the intervention (odds ratio $=1.24$ with $95 \%$ confidence interval $[\mathrm{CI}]=1.21-1.28) .{ }^{26}$ The University of Arizona Institutional Review Board considered this project as exempt.

Benefits and Costs of CMRs Versus Non-CMRs. A key variable in the model was provider acceptance of CMR or nonCMR recommendations by pharmacists. The MMC software tracks each intervention, and these data were extracted for this analysis. Any CMR or non-CMR recommendation approved by a health care provider was assumed to have no associated adverse clinical consequences. Telephone consultation duration and documentation time was randomly collected for 50 calls each for CMRs and non-CMR interventions (Appendix A, available in online article). On average, completing a CMR took 10:13 minutes longer ( $\mathrm{SD}=5: 31$ minutes) than the non-CMR intervention; these variables were used to populate the model. The direct costs of providing CMRs and non-CMR interventions were calculated by multiplying average call duration by the personnel cost for the provider's intervention using a fringe benefit rate of $30 \%$ for personnel. Given that CMRs and nonCMR interventions were conducted in the same office setting, it was assumed that the facilities and administrative costs were identical for each type of intervention and were, therefore, excluded from the analysis.
Clinical Events and Subsequent Costs. Preventable ADEs were considered a potential consequence of providers not accepting pharmacists' CMR or non-CMR recommendations. ADE-related consequences included subsequent hospitalization, emergency department visits, physician visits, additional medications, and death. ${ }^{3,39-41}$ The preventable ADE data were obtained from a cross-sectional observational study conducted with online data collection (Table 1). ${ }^{36}$ Several studies have investigated the costs of ADEs. ${ }^{3,39,42,43}$ However, these studies were conducted in nonambulatory care settings. Instead, the cost of preventable ADEs was estimated from a study conducted in an ambulatory care setting. ${ }^{38}$ These costs were adjusted to January 2012 prices using the Consumer Price Index annual inflation rate. ${ }^{44}$

\section{Base-Case Analysis}

The base-case analysis included the point estimates and plausible ranges for all relevant costs and probabilities used in the model (Table 1). The model is from a health care payer's perspective, so the base-case analysis included direct medical costs associated with providing CMRs or non-CMR interventions and with treating ADEs. The results reported here include the differences in cost between CMRs and non-CMR interventions given that the infrastructure costs were the same for both interventions. The incremental cost-effectiveness ratio (ICER) for differences in cost and effectiveness between CMRs and non-CMR interventions was calculated. TreeAge Pro software (TreeAge Software, Inc., Williamstown, MA) was used to develop the decision model.

\section{Sensitivity Analysis}

Two types of sensitivity analyses were performed: univariate sensitivity analysis and probabilistic sensitivity analysis. All variables were examined using plausible ranges based on SDs 
TABLE 2 Cost-Effectiveness Analysis from Base-Case Analysis and Monte Carlo Simulation

\begin{tabular}{|c|c|c|c|}
\hline Strategy & $\begin{array}{l}\text { Probability of Avoiding } \\
\text { a Preventable ADE } \\
\text { Mean }(95 \% \mathrm{CI})^{\mathrm{a}}\end{array}$ & $\begin{array}{c}\text { Cost Per ADE Prevented } \\
\text { Mean }(95 \% \mathrm{CI})^{\mathrm{a}}\end{array}$ & $\begin{array}{c}\text { Incremental } \\
\text { Cost-Effectiveness Ratio } \\
(95 \% \mathrm{CI})^{\mathrm{a}}\end{array}$ \\
\hline $\mathrm{CMR}^{\mathrm{b}}$ & $0.93(0.91$ to 0.95$)$ & $\$ 192.60$ (\$68.96 to $\$ 391.52)$ & Dominated \\
\hline Non-CMR & $0.94(0.92$ to 0.96$)$ & $\$ 157.02(\$ 46.20$ to $\$ 337.03)$ & $(-\$ 8,356.04$ to $-\$ 3,122.00)$ \\
\hline
\end{tabular}

for the univariate sensitivity analysis (Table 1). For probabilistic sensitivity analysis, beta distributions were used for probability variables, and gamma distributions were used for cost variables. A Monte Carlo simulation was run 10,000 times to determine the mean and SD results for effectiveness (defined as no ADE event rates) and costs for CMRs and non-CMR interventions. A cost-effectiveness acceptability curve (CEAC) was created to demonstrate the likelihood of 1 intervention being cost-effective across a range of willingness-to-pay (WTP) values; WTP values ranged from 0 to 30,000 dollars.

\section{Results}

\section{Base-Case Analysis}

The point-estimate results for non-CMRs were $\$ 157.02$ per 0.94 ADEs prevented, while the CMR results were $\$ 192.60$ per 0.93 ADEs prevented (Appendix Bl, available in online article). The model estimated that non-CMRs saved $\$ 5,377.08$ per ADE prevented compared with CMRs. Given that non-CMRs cost less (\$157.02 vs. \$192.60) and were more effective (0.94 vs. 0.93), they dominated CMRs in this analysis (Table 2).

\section{Sensitivity Analysis Testing}

Table 2 summarizes the probabilistic sensitivity analysis results from the Monte Carlo simulation and provides the mean and 95\% CI results for the costs and ADE events avoided for CMRs and non-CMRs. An overlap in the 95\% CIs was observed between non-CMRs and CMRs for cost and proportion of patients not experiencing ADEs (Appendix B2, available in online article). The Monte Carlo simulation with the costs per ADE avoided were used to generate the cost-effectiveness scatter plot by taking into account the variable distributions for probabilities and costs (Appendix B2). Although there is overlap in the $95 \%$ CIs for costs and ADEs avoided, the majority of points on the scatter plot from non-CMRs show greater effectiveness (i.e., right side of plot) compared with CMRs.

Figure 2 presents the ICER scatter plot. With 10,000 simulation iterations, the mean ICER was $-\$ 5,377.08$ (95\% $\mathrm{CI}=-\$ 8,356.04$ to $-\$ 3,122.00$ ). This figure clearly illustrates the location of the incremental effectiveness data to the right of the vertical axis, indicating that the non-CMR interventions resulted in a larger number of patients without ADEs (i.e., more successful interventions). In addition, all points on the ICER scatter plot of non-CMRs versus CMRs were in the lower right quadrant of the plane. That is, non-CMRs led to a greater proportion of ADEs avoided (i.e., more successful interventions) at a lower cost than CMRs. The CEAC from the Monte Carlo simulation demonstrates that in $100 \%$ of scenarios, non-CMRs were the most likely cost-effective option when compared with CMRs, regardless of the health plan's willingness to pay (Appendix C, available in online article).

\section{Univariate Sensitivity Analyses}

Univariate or one-way sensitivity analyses of the most influential variables are included in a tornado diagram (Figure 3). In the diagram, each bar represents the impact of uncertainty an individual parameter has on the results by varying the values within their plausible ranges (Table 1). The variables with the greatest impact in this analysis were the cost of treating a preventable ADE and incremental costs of providing CMRs (over non-CMR interventions; Figure 3). However, the model was robust-no individual variable changed the conclusion that non-CMRs were most cost-effective, since the ICERs remained in the positive area.

\section{Discussion}

The point-estimate results indicate that non-CMR interventions were more effective and less costly as a method for preventing ADEs when compared with CMRs. In addition, the ICER data suggest that performing non-CMRs could save an additional $\$ 5,377.08$ for every ADE prevented. However, the results were sensitive to the cost of treating a preventable ADE. The Monte Carlo simulation of 10,000 scenarios revealed that non-CMRs dominated CMRs in almost all scenarios and was supported by the CEAC graph.

The main driving force behind the cost savings in favor of non-CMRs over CMRs was the likelihood of medication changes, since it was the only variable that had different values between the 2 interventions. There are several potential explanations for this observation. First, prescribers may pay closer attention when recommendations are focused and specific. The information provided by CMRs, although useful, may cause prescribers to be less prone to action because they 
Cost-Effectiveness of Comprehensive Medication Reviews Versus Noncomprehensive Medication Review Interventions and Subsequent Successful Medication Changes in a Medicare Part D Population

\section{FIGURE 2 Incremental Cost-Effectiveness Scatter Plot of Non-CMR Interventions Versus CMRs}

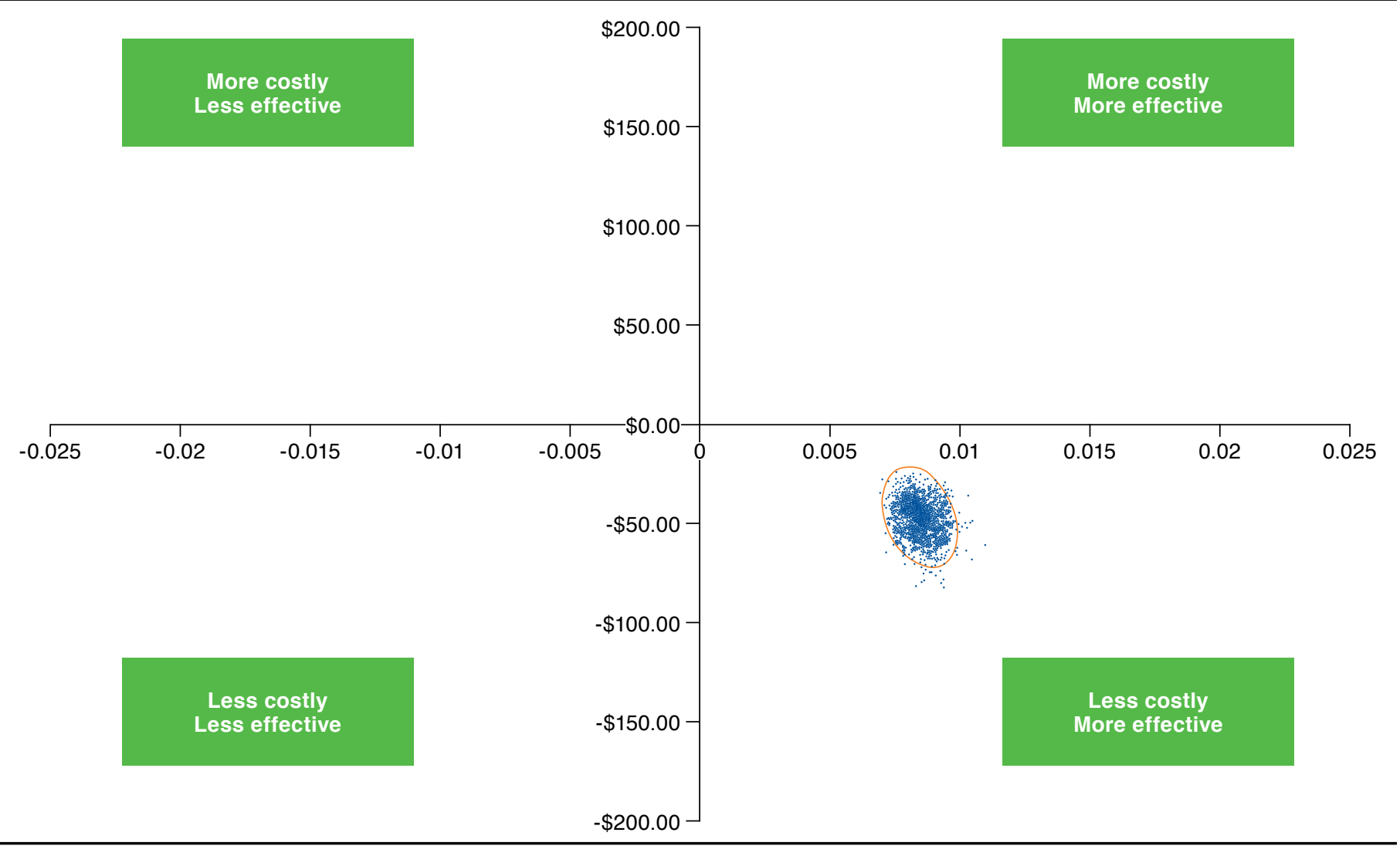

$C M R=$ comprehensive medication review; non-CMR=noncomprehensive medication review.

feel reluctant to make multiple medication regimen changes at the same time ${ }^{45}$ As a result, prescribers may be more likely to accept recommendations resulting from non-CMRs. Thus, by prioritizing and suggesting the most important MRPs, this could improve the likelihood of gaining the prescribers' acceptance for non-CMR interventions.

Second, patients with chronic illness struggle with managing their day-to-day and long-term health. Health information, the health care system, and medications regimens are complex and confusing. By tailoring straightforward, more targeted approaches to addressing identified DRPs, it is possible to simplify provider-patient communication and patient engagement materials to help improve adherence, patient outcomes, and overall health. The randomized trial by Kreuter and Strecher (1996) indicates that individually tailored messages are almost $20 \%$ more likely to change at least 1 behavior. ${ }^{46}$

The MMC's innovative software identifies MPRs (i.e., alerts), classifies them into different levels by complexity and urgency, and then assigns the individual case to receive one of several intervention strategies: CMR exclusively, CMR and non-CMR options, or non-CMR options only (e.g., short calls, letters, or direct provider outreach). Thus, for those beneficiaries who require fewer medication changes, performing non-CMRs may be equal to or more effective than CMRs.

Non-CMRs may also help improve the efficiency of MTM programs, given that $90 \%$ of all medication changes occurred in the non-CMR group. Furthermore, almost $90 \%$ of Medicare Part D beneficiaries who met the criteria for a CMR did not participate in this type of intervention; instead, they received non-CMR interventions. Non-CMR options require, on average, 10 minutes less per beneficiary than CMRs, thus enabling the MMC staff to perform additional non-CMRs per day, providing more opportunities to detect MRPs and increase the potential to prevent subsequent ADEs. This MTM program has mechanisms in place to ensure that beneficiaries receive appropriate interventions for their MRPs. However, for some MTM programs that choose to provide only CMRs, $90 \%$ of the aforementioned beneficiaries would have received no intervention.

It is worth noting that CMS encourages intervention strategies for MRPs identified during required quarterly TMRs for 


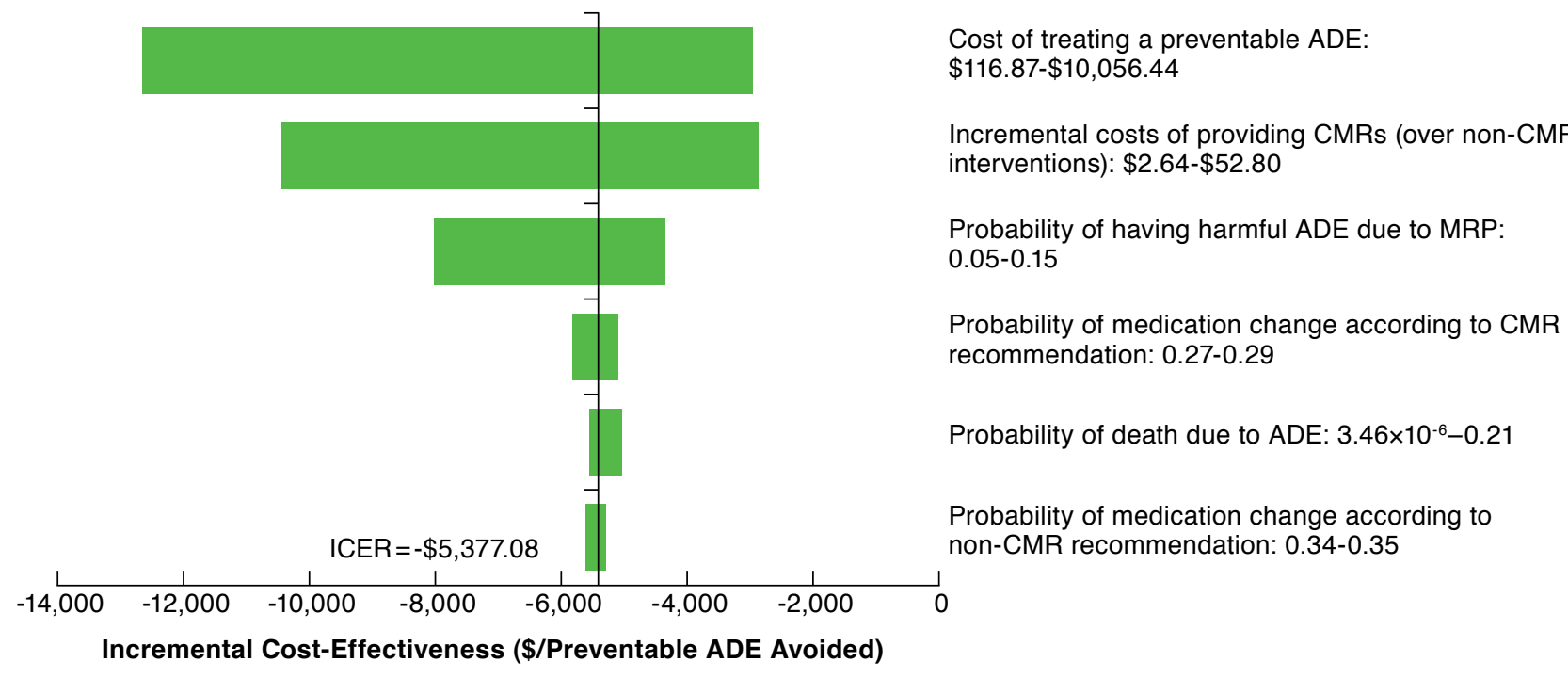

$A D E=$ adverse drug effect; $C M R=$ comprehensive medication review; ICER =incremental cost effectiveness ratio; $M R P=$ medication-related problem; non- $C M R=$ noncomprehensive medication review.

Medicare Part D beneficiaries who meet minimum qualification criteria (i.e., multiple chronic health conditions and multiple medications). However, CMS only uses the annual percentage of patients who receive CMRs as an MTM performance metric. Thus, we know from watching measurement history that metrics drive performance. For example, a managed care organization implemented processes to ensure patients received beta-blocker therapy after the National Committee for Quality Assurance began measuring whether patients experiencing a myocardial infarction had received the respective treatment. ${ }^{47}$ Ultimately, the new metric prompted response, and more than $90 \%$ of patients who had a myocardial infarction received betablocker treatment. Similarly, the current study suggests the need for inclusion of MTM intervention strategies as measures in the MTM performance metrics (i.e., star ratings for Medicare Advantage and prescription drug plans proposed by CMS) to incentivize interventions to reduce MRPs and, ultimately, to reduce the ADE-related costs for managed care organizations. These study results suggest that MTM programs have an opportunity to improve their efficiency in delivering services. Moreover, this may serve as a driving force behind the expansion of MTM services.

\section{Limitations}

This analysis has several important limitations. The lack of availability of complete medical records prevents the ability to determine other clinical outcomes (e.g., adherence, immu- nization rates, and health condition monitoring) that MTM interventions aim to improve, as well as long-term outcomes such as mortality. Although a difference in point estimate of avoiding a preventable ADE was examined in this study, it does not imply a difference in clinically meaningful outcomes. The innate nature of a retrospective analysis prevents randomization between CMR and non-CMR groups and so is a study limitation. Nonrandomization introduces the potential for selection bias or systematic error given that other confounders may be present that could have an impact on the dependent and independent variables. Also, patients who agreed to have a CMR may differ significantly with regard to their characteristics (e.g., demographics, chronic illness history, and health literacy) from those who received non-CMR services. There is the potential for threats to external validity given that CMRs and non-CMR interventions were conducted at a single facility. Therefore, this raises the issue whether the results are generalizable to other facilities providing MTM services. Additionally, all consultations were performed via telephone, thus, these results may not be generalizable to MTM programs that offer face-to-face consultations. Lastly, the published literature regarding written prescribing errors may have some differences compared with pharmacist telephone interventions regarding DRPs.

\section{Conclusions}

This cost-effectiveness study suggests that non-CMR interventions are less costly and more effective than CMRs in preventing ADEs and reducing health care costs, when viewed from a health 


\section{Cost-Effectiveness of Comprehensive Medication Reviews Versus Noncomprehensive Medication Review Interventions and Subsequent Successful Medication Changes in a Medicare Part D Population}

care payer's perspective. The Monte Carlo simulation of 10,000 scenarios found that non-CMRs dominated CMRs in almost all scenarios. Results were most sensitive to cost of treating a preventable ADE. These results suggest the need for inclusion of non-CMR interventions in the metrics evaluating Medicare Part D MTM programs. Future research is warranted to further evaluate non-CMR interventions in other populations and to examine other potential beneficial effects such as improvement in medication adherence. In summary, non-CMRs show promise as a viable method for pharmacists and patients to address MRPs, reduce ADEs, and improve patient-related health outcomes.

\section{Authors}

CHANADDA CHINTHAMMIT, BSPharm, MS, is a PhD Student, Department of Pharmaceutical Sciences, and ROSE MARTIN, PharmD, is Director, Medication Management Center, College of Pharmacy, University of Arizona, Tucson. EDWARD P. ARMSTRONG, PharmD, is Professor Emeritas; ANN M. TAYLOR, MPH, MCHES, is Program Manager, HOPE Center; and TERRI WARHOLAK, RPh, PhD, is Associate Professor, Department of Pharmacy Practice and Science, College of Pharmacy, University of Arizona, Tucson. KEVIN BOESEN, PharmD, is Chief Executive Officer, SinfoniaRx, Tucson, Arizona.

AUTHOR CORRESPONDENCE: Chanadda Chinthammit, BSPharm, MS, Dept. of Pharmaceutical Sciences, University of Arizona College of Pharmacy, 1295 N. Martin Ave., Tucson, Arizona 85721. Tel.: 520.743.6271; E-mail: chinthammit@pharmacy.arizona.edu.

\section{DISCLOSURES}

This research was conducted by University of Arizona College of Pharmacy, Tucson, Arizona, without external funding. Boesen is an employee of SinforniaRx.

Concept and design were performed by Chinthammit, Boesen, Martin, and Warholak. Data were collected by Chinthammit, Armstrong, and Martin, with help from Boesen and Warholak. Data were interpreted by Warholak, Armstrong, and Chinthammit. Chinthammit, Armstrong, and Warholak wrote the manuscript with help from Boesen and Martin. The manuscript was revised by Taylor, Warholak, and Chinthammit, with help from Armstrong, Boesen, and Martin.

\section{REFERENCES}

1. Kohn LT, Corrigan JM, Donaldson MS, eds. To Err Is Human: Building a Safer Health System. National Academies Press; 2000.

2. Kass B. Reducing and preventing adverse drug events to decrease hospital costs. Research in Action, Issue 1. March 2001. Agency for Healthcare Research and Quality, Rockville, MD. Available at: http://archive.ahrq.gov/ research/findings/factsheets/errors-safety/aderia/ade.html. Accessed March $14,2015$.

3. Johnson JA, Bootman JL. Drug-related morbidity and mortality. A cost-ofillness model. Arch Intern Med. 1995;155(18):1949-56.

4. Chrischilles EA, Segar ET, Wallace RB. Self-reported adverse drug reactions and related resource use. A study of community-dwelling persons 65 years of age and older. Ann Intern Med. 1992;117(8):634-40.
5. Hanlon JT, Weinberger M, Samsa GP, et al. A randomized, controlled trial of a clinical pharmacist intervention to improve inappropriate prescribing in elderly outpatients with polypharmacy. Am J Med. 1996;100(4):428-37.

6. Hohl CM, Dankoff J, Colacone A, Afilalo M. Polypharmacy, adverse drugrelated events, and potential adverse drug interactions in elderly patients presenting to an emergency department. Ann Emerg Med. 2001;38(6):666-71.

7. Nguyen JK, Fouts MM, Kotabe SE, Lo E. Polypharmacy as a risk factor for adverse drug reactions in geriatric nursing home residents. Am J Geriatr Pharmacother. 2006;4(1):36-41.

8. Rollason V, Vogt N. Reduction of polypharmacy in the elderly. Drugs Aging. 2003;20(11):817-32

9. Osterberg L, Blaschke T. Adherence to medication. N Engl J Med. 2005;353(5):487-97.

10. Marks JR, Schectman JM, Groninger H, Plews-Ogan ML. The association of health literacy and socio-demographic factors with medication knowledge. Patient Educ Couns. 2010;78(3):372-76.

11. Hammons T, Piland NF, Small SD, Hatlie MJ, Burstin HR. Ambulatory patient safety: what we know and need to know. J Ambul Care Manage. 2003;26(1):63-82

12. Tamblyn R, Laprise R, Hanley JA, et al. Adverse events associated with prescription drug cost-sharing among poor and elderly persons. JAMA. 2001;285(4):421-29.

13. O'Dell KM, Kucukarslan SN. Impact of the clinical pharmacist on readmission in patients with acute coronary syndrome. Ann Pharmacother. 2005;39(9):1423-27.

14. Kucukarslan SN, Peters M, Mlynarek M, Nafziger DA. Pharmacists on rounding teams reduce preventable adverse drug events in hospital general medicine units. Arch Intern Med. 2003;163(17):2014-18.

15. Krska J, Cromarty JA, Arris F, et al. Pharmacist-led medication review in patients over 65: a randomized, controlled trial in primary care. Age Ageing 2001;30(3):205-11.

16. Doucette WR, McDonough RP, Klepser D, McCarthy R. Comprehensive medication therapy management: identifying and resolving drug-related issues in a community pharmacy. Clin Ther. 2005;27(7):1104-11.

17. Galindo C, Olivé M, Lacasa C, et al. Pharmaceutical care: pharmacy involvement in prescribing in an acute-care hospital. Pharm World Sci. 2003;25(2):56-64

18. Stolar MW, Hoogwerf BJ, Gorshow SM, Boyle PJ, Wales DO. Managing type 2 diabetes: going beyond glycemic control. J Manag Care Pharm. 2008;14(5 Suppl B):S2-19. Available at: http://www.amcp.org/data/jmcp/ JMCPSuppBJune08Web.pdf.

19. Spence MM, Makarem AF, Reyes SL, et al. Evaluation of an outpatient pharmacy clinical services program on adherence and clinical outcomes among patients with diabetes and/or coronary artery disease. J Manag Care Pharm. 2014;20(10):1036-45. Available at: http://www.amcp.org/WorkArea/ DownloadAsset.aspx?id=18574.

20. Perera PN, Guy MC, Sweaney AM, Boesen KP. Evaluation of prescriber responses to pharmacist recommendations communicated by fax in a medication therapy management program (MTMP). J Manag Care Pharm. 2011;17(5):345-54. Available at: http://www.amcp.org/WorkArea/ DownloadAsset.aspx?id=9737.

21. The Medicare Prescription Drug, Improvement, and Modernization Act of 2003. Public Law 108-173. December 8, 2003. Available at: http://www. gpo.gov/fdsys/pkg/PLAW-108publl73/content-detail.html. Accessed March $14,2015$.

22. Barnett MJ, Frank J, Wehring H, et al. Analysis of pharmacist-provided medication therapy management (MTM) services in community pharmacies over 7 years. J Manag Care Pharm. 2009;15(1):18-31. Available at: http:// www.amcp.org/data/jmcp/018-031.pdf. 
23. Oberlander J. Through the looking glass: the politics of the Medicare Prescription Drug, Improvement, and Modernization Act. J Health Polit Policy Law. 2007;32(2):187-219.

24. Lounsbery JL, Green CG, Bennett MS, Pedersen CA. Evaluation of pharmacists' barriers to the implementation of medication therapy management services. J Am Pharm Assoc. 2008;49(1):51-58.

25. Watkins JL, Landgraf A, Barnett CM, Michaud L. Evaluation of pharmacist-provided medication therapy management services in an oncology ambulatory setting. J Am Pharm Assoc (2003). 2012;52(2):170-74.

26. Buhl AK, Augustine J, Chinthammit C, Boeson KP. Comparison of the number of patients with medication changes following comprehensive versus targeted medication reviews in a medication therapy management program. Poster presented at: American Pharmacists Association (APhA) 2014 Annual Meeting \& Exposition; March 28-31, 2014; Orlando, FL.

27. Tudor C. CY 2013 Medication therapy management program guidance and submission instructions. Memorandum. April 10, 2012. Available at: http://www.cms.gov/Medicare/Prescription-Drug-Coverage/ PrescriptionDrugCovGenIn/Downloads/Memo-Contract-Year-2013Medication-Therapy-Management-MTM-Program-Submission-v041012.pdf. Accessed March 14, 2015.

28. Schommer JC, Planas LG, Johnson KA, Doucette WR. Pharmacistprovided medication therapy management (part 1): provider perspectives in 2007. J Am Pharm Assoc (2003). 2008;48(3):354-63.

29. Schommer JC, Planas LG, Johnson KA, Doucette WR. Pharmacistprovided medication therapy management (part 2): payer perspectives in 2007. J Am Pharm Assoc (2003). 2008;48(4):478-86.

30. Isetts BJ, Schondelmeyer SW, Artz MB, et al. Clinical and economic outcomes of medication therapy management services: the Minnesota experience. J Am Pharm Assoc (2003). 2008;48(2):203-11.

31. Knoell DL, Pierson JE, Marsh CB, Allen JN, Pathak DS. Measurement of outcomes in adults receiving pharmaceutical care in a comprehensive asthma outpatient clinic. Pharmacotherapy. 1998;18(6):1365-74.

32. Ramalho de Oliveira D, Brummel AR, Miller DB. Medication therapy management: 10 years of experience in a large integrated health care system. J Manag Care Pharm. 2010;16(3):185-95. Available at: http://www.amcp.org/ data/jmcp/185-195.pdf.

33. Cranor CW, Christensen DB. The Asheville Project: short-term outcomes of a community pharmacy diabetes care program. J Am Pharm Assoc (2003). 2012;52(6):838-50

34. Cranor CW, Bunting BA, Christensen DB. The Asheville Project: longterm clinical and economic outcomes of a community pharmacy diabetes care program. J Am Pharm Assoc (2003). 2003;43(2):173-84.
35. Centers for Medicare \& Medicaid Services. 2012 Plan MTM program eligibility information. Available at: http://www.cms.gov/Medicare/ Prescription-Drug-Coverage/PrescriptionDrugCovContra/MTM.html. Accessed March 14, 2015.

36. Kuo GM, Touchette DR, Marinac JS. Drug errors and related interventions reported by United States clinical pharmacists: the American College of Clinical Pharmacy practice-based research network medication error detection, amelioration and prevention study. Pharmacotherapy. 2013;33(3):253-65.

37. Bates DW, Boyle DL, Vander Vliet MB, Schneider J, Leape L. Relationship between medication errors and adverse drug events. J Gen Intern Med. 1995;10(4):199-205

38. Field TS, Gilman BH, Subramanian S, Fuller JC, Bates DW, Gurwitz JH. The costs associated with adverse drug events among older adults in the ambulatory setting. Med Care. 2005;43(12):1171-76.

39. Ernst FR, Grizzle AJ. Drug-related morbidity and mortality: updating the cost-of-illness model. J Am Pharm Assoc (Wash). 2001;41(2):192-99.

40. Chinthammit C, Armstrong EP, Warholak TL. A cost-effectiveness evaluation of hospital discharge counseling by pharmacists. J Pharm Pract. 2012;25(2):201-08.

41. Bates DW, Spell N, Cullen DJ, et al. The costs of adverse drug events in hospitalized patients. Adverse Drug Events Prevention Study Group. JAMA 1997;277(4):307-11

42. Prince B, Goetz C, Rihn T, Olsky M. Drug-related emergency department visits and hospital admissions. Am J Health Syst Pharm. 1992;49(7):1696-700.

43. Tafreshi MJ, Melby MJ, Kaback KR, Nord TC. Medication-related visits to the emergency department: a prospective study. Ann Pharmacother. 1999;33(12):1252-57.

44. U.S. Department of Labor, Bureau of Labor Statistics. Consumer Price Index. Available at: http://www.bls.gov/cpi/home.htm. Accessed March 14, 2015

45. Vinks TH, Egberts TC, de Lange TM, de Koning FH. Pharmacist-based medication review reduces potential drug-related problems in the elderly. Drugs Aging. 2009;26(2):123-33.

46. Kreuter MW, Strecher VJ. Do tailored behavior change messages enhance the effectiveness of health risk appraisal? Results from a randomized trial. Health Educ Res. 1996;11(1):97-105.

47. National Committee for Quality Assurance. The state of health care quality 2007. Available at: http://www.ncqa.org/Portals/0/Publications/ Resource\%20Library/SOHC/SOHC_07.pdf. Accessed March 14, 2015.

48. U.S. Department of Labor, Bureau of Labor Statistics. Occupational outlook handbook. 2013. Available at: http://www.bls.gov/ooh/. Accessed March $14,2015$. 
Cost-Effectiveness of Comprehensive Medication Reviews Versus Noncomprehensive Medication Review Interventions and Subsequent Successful Medication Changes in a Medicare Part D Population

\section{APPENDIX A Characteristics of Medication Management Center Call Duration}

\begin{tabular}{l|c|c}
\hline Model Parameter & Mean (SD) & Reference \\
\hline Time to perform CMRs (minutes) & $23: 25(11: 18)$ & MMC internal data \\
\hline Time to perform non-CMR interventions (minutes) & $13: 13(7: 28)$ & MMC internal data \\
\hline Additional time to perform CMRs (over non-CMRs; minutes) & $10: 12(5: 31)$ & MMC internal data \\
\hline Average pharmacist hourly wage & $\$ 55.27$ & U.S. Department of Labor ${ }^{48}$ \\
\hline MMC pharmacist fringe benefit rate & $30 \%$ & MMC internal data \\
\hline CMR comprehensive medication review: MMC = Medication Management Center: non-CMR=noncomprehensive medication review: SD standard deviation.
\end{tabular}

$C M R=$ comprehensive medication review; $M M C=$ Medication Management Center; non-CMR=noncomprehensive medication review; $S D=$ standard deviation.

\section{APPENDIX B Cost-Effectiveness Point Estimates and Scatter Plots for CMRs and Non-CMR Interventions}

\section{Cost-Effectiveness Point Estimates}

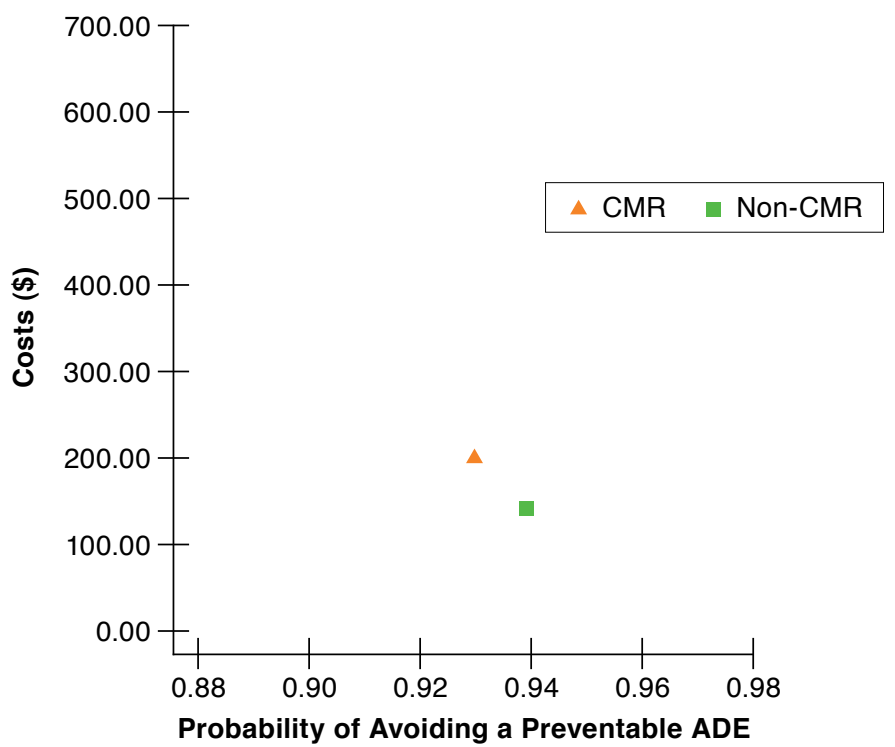

\section{Cost-Effectiveness Scatter Plots}

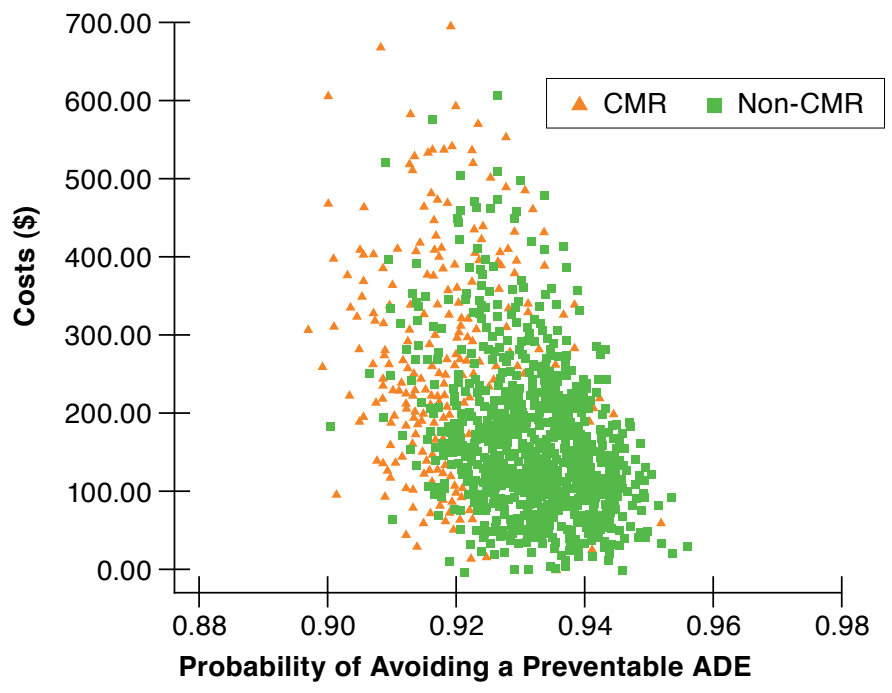

$A D E=$ adverse drug event $; C M R=$ comprehensive medication review; non-CMR=noncomprehensive medication review.

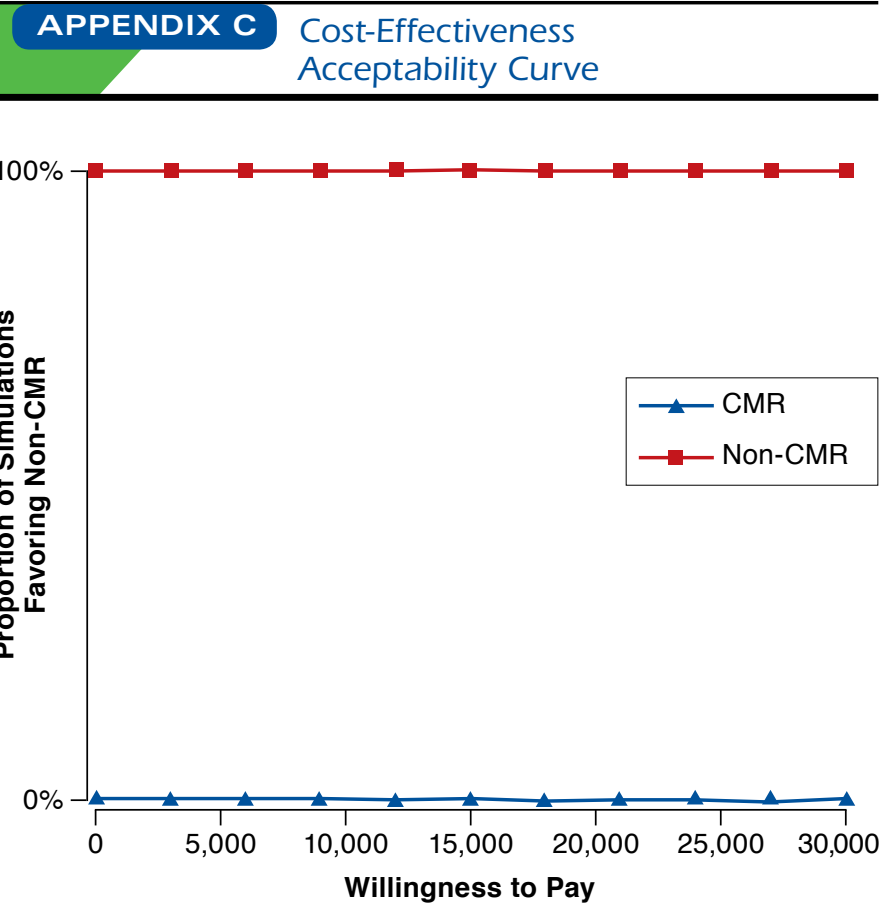

$C M R=$ comprehensive medication review; non $C M R=$ noncomprehensive medication review. 